\title{
IMPORTANCIA DE LAS ESTRUCTURAS GEOLÓGICAS EN EL MODELO CONCEPTUAL DEL SISTEMA ACUÍFERO GUARANÍ -AREA URUGUAYA
}

\author{
Jorge Montaño Xavier ${ }^{1}$ \\ Ernani Francisco da Rosa Filho ${ }^{2}$ \\ Eduardo Chemas Hindi ${ }^{2}$ \\ Hugo Cicalese ${ }^{3}$ \\ Mauricio Montaño Gutierrez ${ }^{4}$ \\ Sergio Gagliardi Urtasun ${ }^{4}$
}

\section{Introducción}

El Sistema Acuífero Guaraní (SAG) es un recurso hídrico subterráneo casi único a nivel mundial por ser unas de las reservas hídricas más importantes del mundo .Es compartido por los cuatro países que actualmente integran el MERCOSUR, extendiéndose en sus territorios por alrededor de $1.400 .000 \mathrm{~km}^{2}$ y con un potencial hídrico del orden de $40.000 \mathrm{~km}^{3}$. En gran parte del área presenta condiciones de termalismo y surgencia. Tradicionalmente se establecía que este sistema acuífero representaba un cuerpo homogéneo cuyas variaciones se deberían principalmente a su espesor y a cambios litológicos que no eran significativos.

El ejemplo que presentamos corresponde al SAG en el Uruguay y parte de Argentina, área que forma parte del limite sur del SAG y de la cuenca sedimentaria. Esta zona se caracteriza por su gran heterogeneidad producto de la actividad tectónica típica de borde de cuenca intracratónica, con fracturamiento, fallas, desplazamientos de bloques, presencia de filones, etc. Estas condiciones influyen en las dimensiones y la dinámica del sistema acuífero, lo que lleva a separar el área en ámbitos hidrogeológicamente diferentes.

En Uruguay, el Acuífero Guaraní forma parte de la denominada Cuenca Sedimentaria Norte, que se desarrolla en el
Noreste y Centro-Norte del Uruguay, ocupando un área de aproximadamente $100.000 \mathrm{~km}^{2}$. En esta cuenca sedimentaria el Acuífero Guaraní ocupa una superficie aproximada a los $43.000 \mathrm{~km}^{2}$. En la mayoría del área se encuentra protegido por una capa de basalto de la Formación Arapey $\left(38.000 \mathrm{~km}^{2}\right)$, cuyo espesor máximo es de $1200 \mathrm{~m}$. El resto corresponde a la zona de afloramientos situada en el centro norte del País.

\section{Desarrollo}

Se define el típico Sistema Acuífero Guaraní al conjunto sedimentario de edad triásica - jurásica constituido por los niveles de media a alta permeabilidad del Grupo Batoví Dorado integrado por las formaciones Rivera, Tacuarembó y Cuchilla de Ombú y de las formaciones Buena Vista y Yaguarí de edad triásica.

El Sistema Acuífero Guaraní SAG en este contexto está constituido principalmente por una sucesión de areniscas de origen desértico y fluvio-lacustre de edad Triásico Jurásica, con espesores que pueden variar desde pocos metros hasta casi $200 \mathrm{~m}$, totalmente saturados de agua. Gran parte de esa superficie está cubierta y protegida por extensos y potentes derrames basálticos, que constituyen su techo, en la zona oeste del País, limítrofe con Argentina (Figura 1). Hacia el centro del País a una distancia de

\footnotetext{
1 (montanox@movinet.com.uy) - Universidad de la República - Facultad de Ciencias - Departamento de Geología (Uruguay)

2 (ernani@geología.ufpr.br); (hindi@geología.ufpr.br) -Universidad Federal de Paraná (Brasil).

3 (cicalese@conectate.com.uy) - Dirección Nacional de Minería y Geología (Uruguay).

4 (mmontano@internet.com.uy); (sgagliar@internet.com.uy) - Estudiantes de la Universidad de la República - Facultad de Ciencias - Departamento de Geología (Uruguay)
} 
200 km desde el Río Uruguay las areniscas afloran principalmente en la zona norte zonas de Tranqueras y ciudad de Rivera

\section{Estructura del SAG}

En una primera instancia, se divide el sistema en función de sus características geológicas en dos ámbitos:

\subsection{Zona Confinada}

La zona confinada es la parte del acuífero que se encuentra cubierta por un techo de basalto con espesores de hasta $1200 \mathrm{~m}$ y ocupa un área aproximada a los $38.000 \mathrm{~km}^{2}$ al oeste del país (Figura 1).
EI SAG típico se apoya sobre sedimentos pérmicos de media a baja permeabilidad, cuyo ambiente de formación es marino, lo que condiciona la calidad del agua enriqueciéndola en sales, constituyendo el segundo sistema hidrogeológico del área.

Estos dos sistemas no se presentan en toda la cuenca simultáneamente sino que están condicionados a los paleoambientes de sedimentación y paleotectonica. Sin embargo en función de sus áreas de desarrollo 0 predominio se pueden establecer en forma esquemática dos zonas: Norte y Sur.

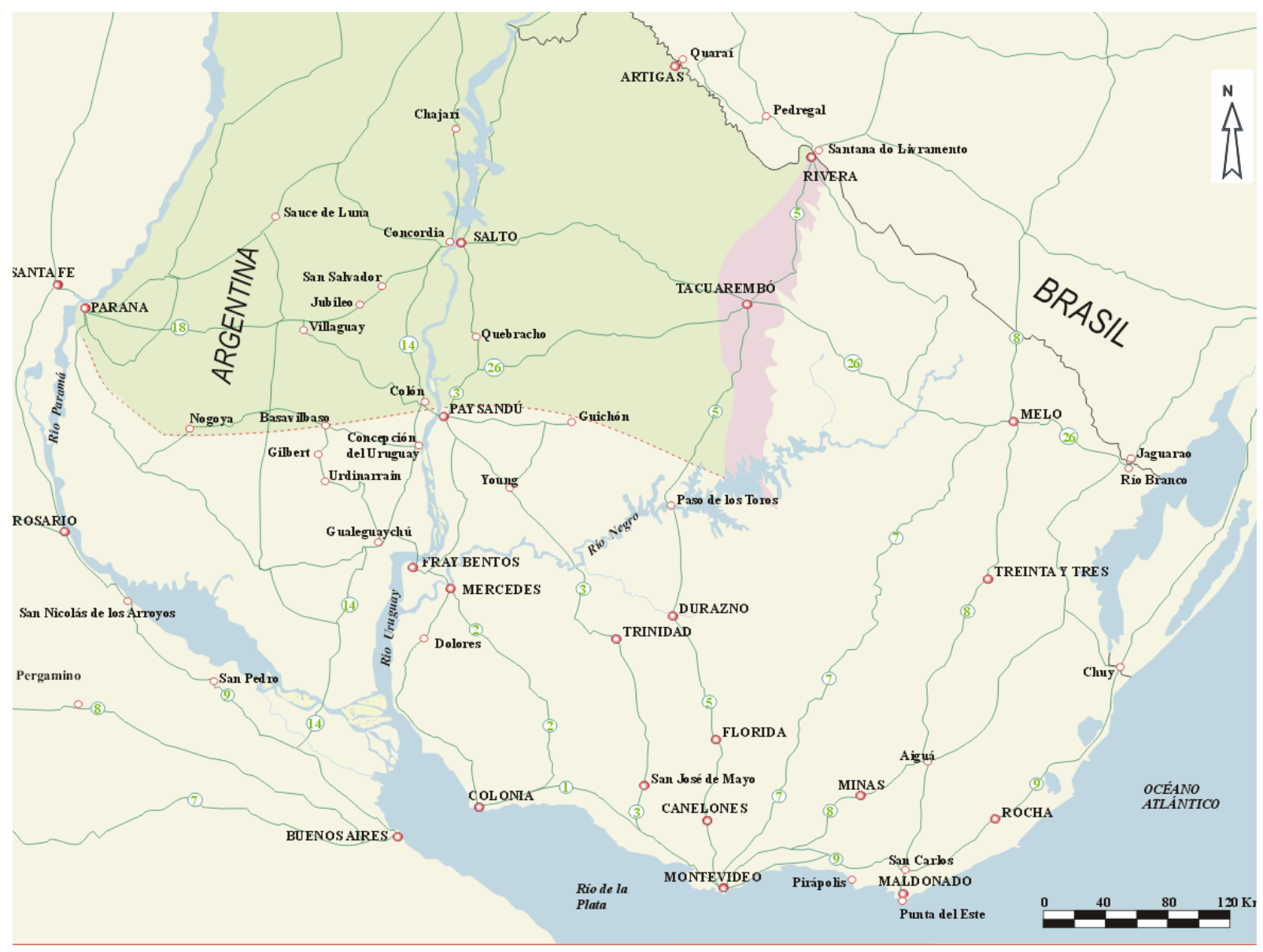

REFERENCIAS

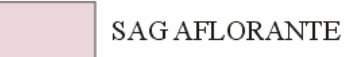

SAG Confinado. Cubierto por basaltos y sedimentos cretácicos hasta cuaternarios.

Figura 1. Mapa general: Zona aflorante y Zona confinada del Sistema Acuífero Guaraní Area Uruguaya

\subsubsection{Zona Norte}

Hacia el norte de la cuenca el SAG se presenta en su mayoría del área cubierto, aunque existe en el límite con Brasil (Ciudades de Artigas y Quaraí) una "ventana" de afloramiento del acuífero. 
En esta región presenta su mayor dimensión (figura 2) y se comporta generalmente en forma independiente del sistema hidrogeológico de origen pérmico. Decimos generalmente porque todavía no se ha comprobado en esta zona ninguna conexión entre ambos sistemas, aunque el área presenta una gran actividad tectónica, (fallas, dislocamiento de bloques, presencia de filones de diabasa, etc) que podrían ser conductores entre estos cuerpos de aguas.

Los pozos se presentan con caudales de surgencia 90 a $340 \quad \mathrm{~m}^{3} / \mathrm{h}$ con transmisividades entre 300 a $400 \mathrm{~m}^{2} /$ día. Se destaca que la mayoría de los pozos son utilizados para abastecimiento de centros turísticos termales. La calidad del agua se clasifica como bicarbonatada sódica.

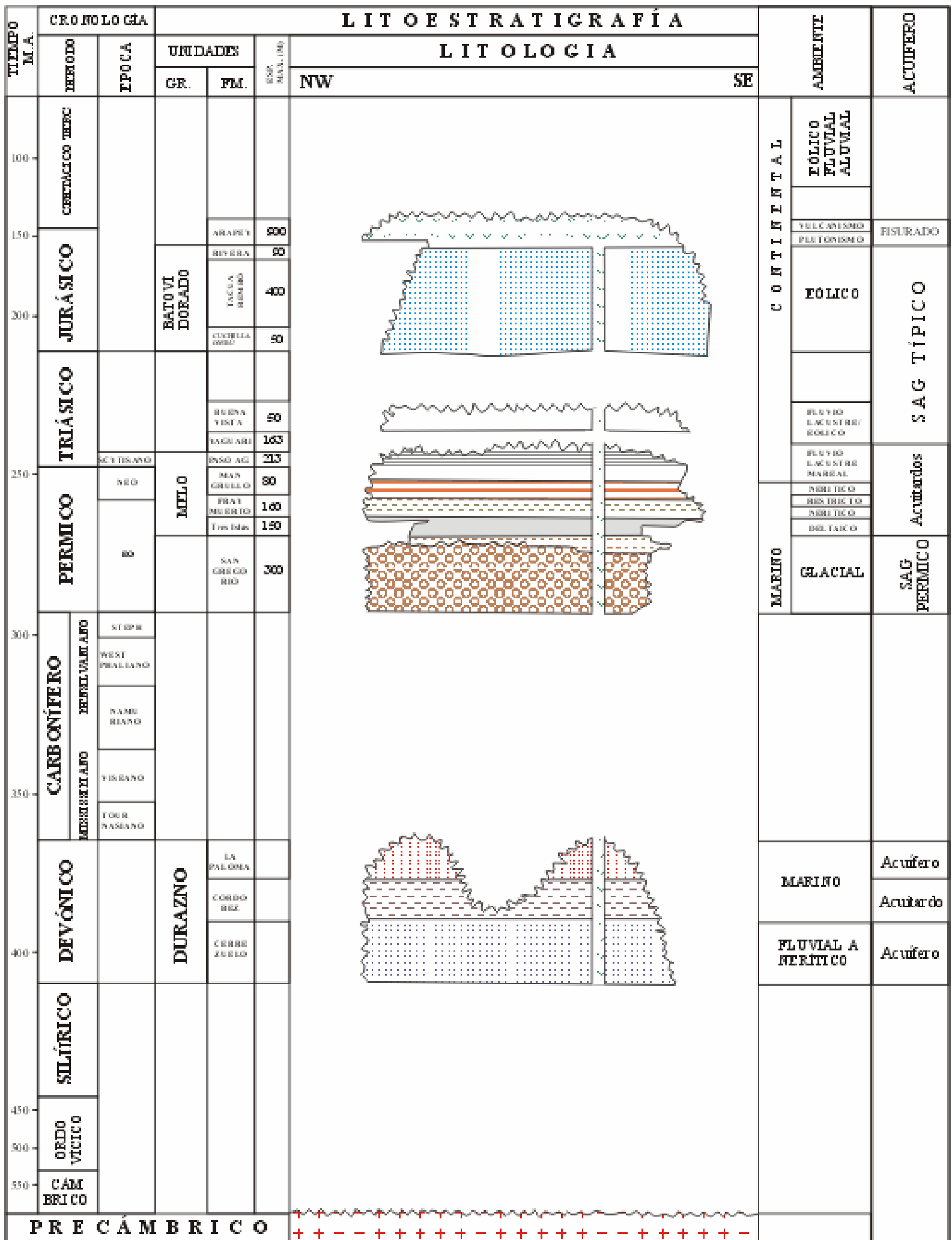




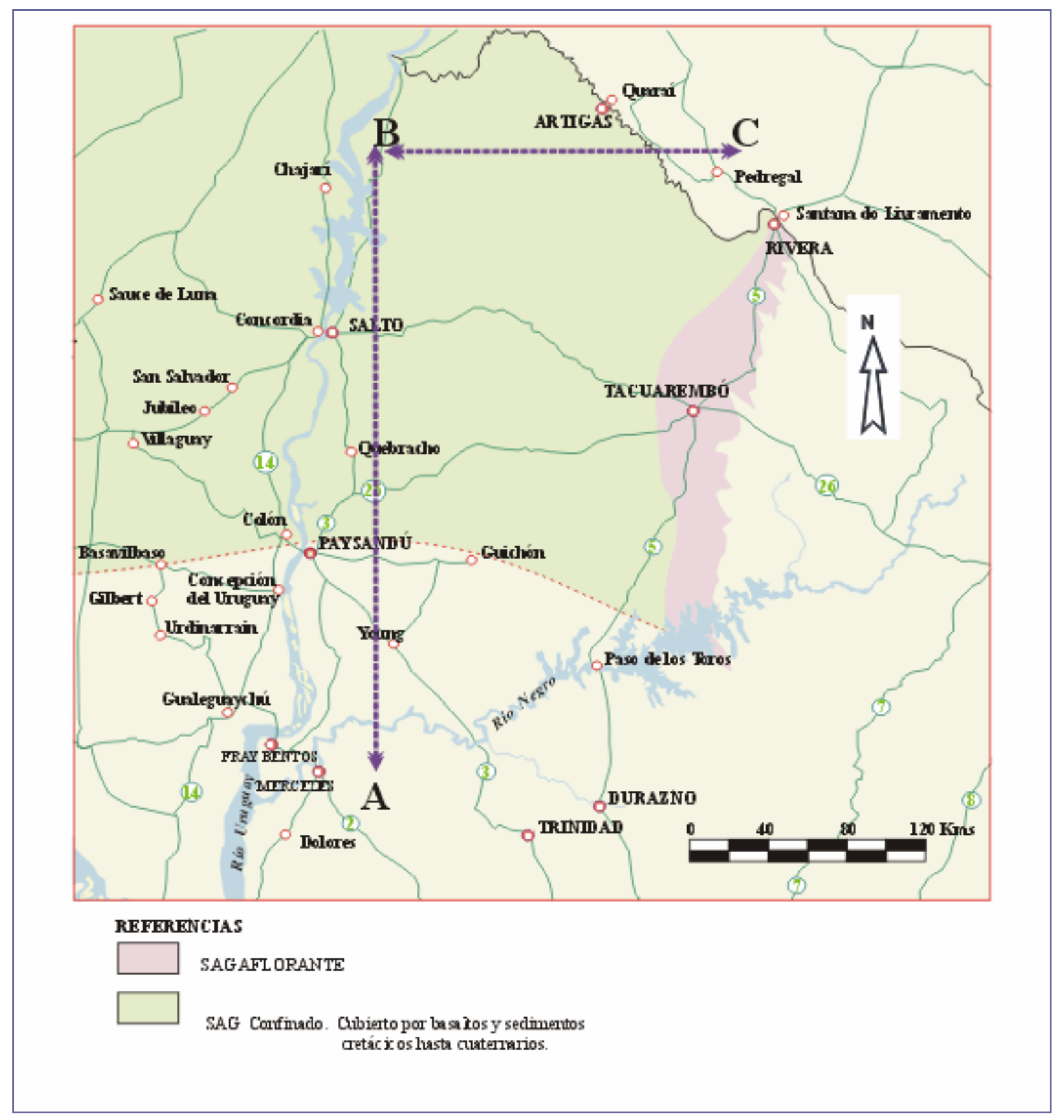

Figura 3. Ubicación de los cortes 


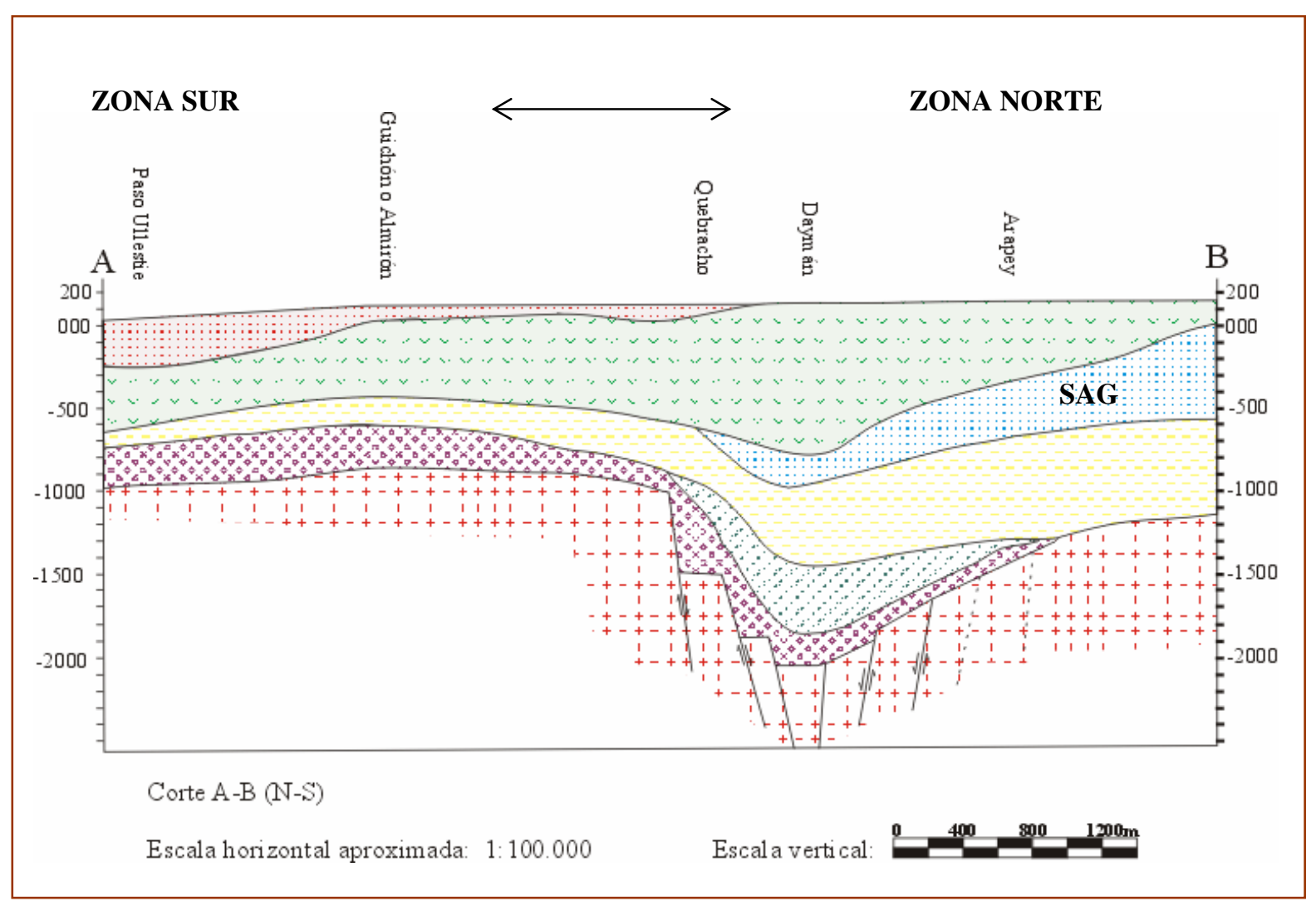

Figura 5. Referencias. Corte N-S y E-W. Sistema Acuífero Guaraní - Area Uruguaya

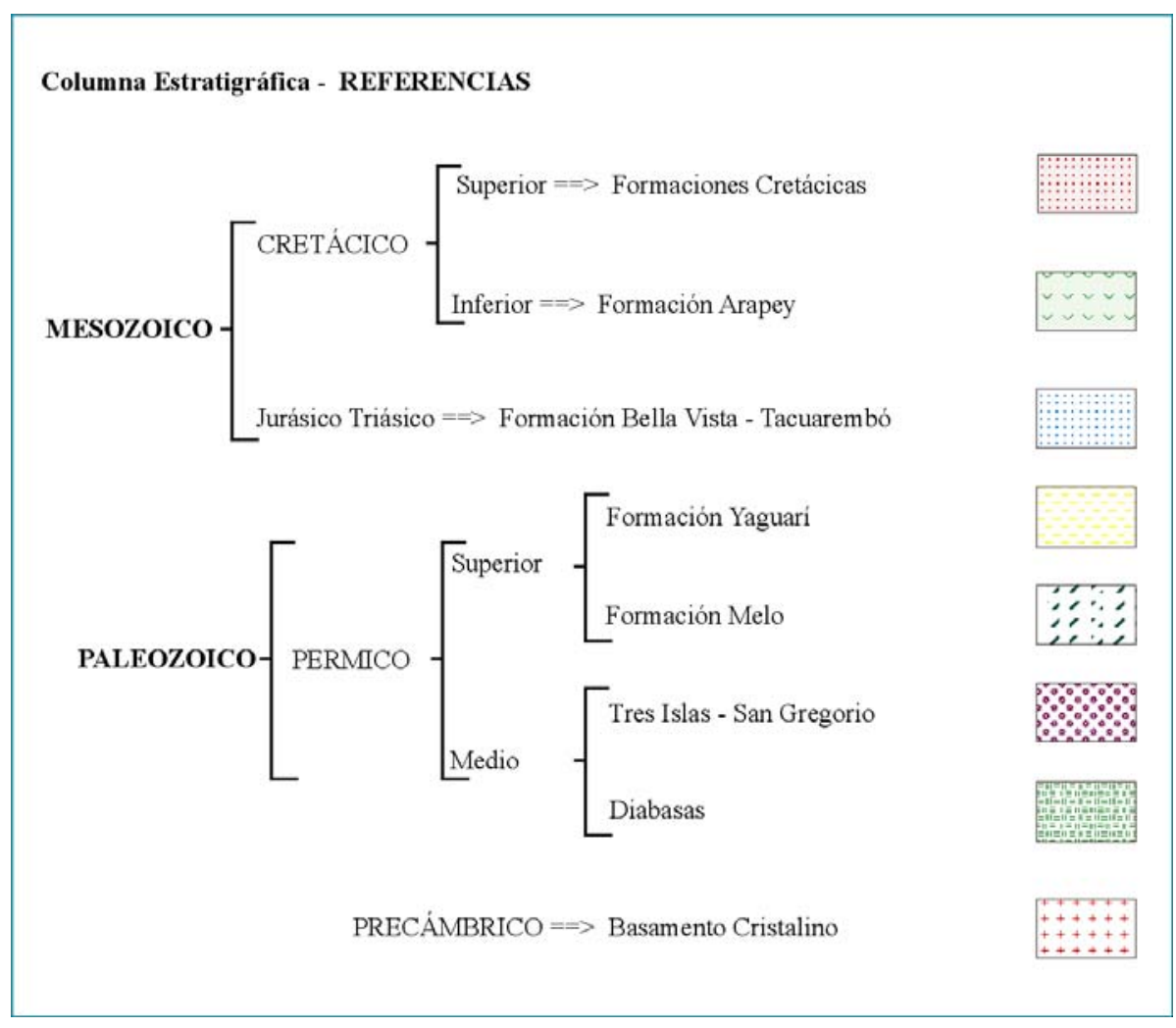

Figura 6. Corte E-W. Sistema Acuífero Guaraní - Area Uruguaya 


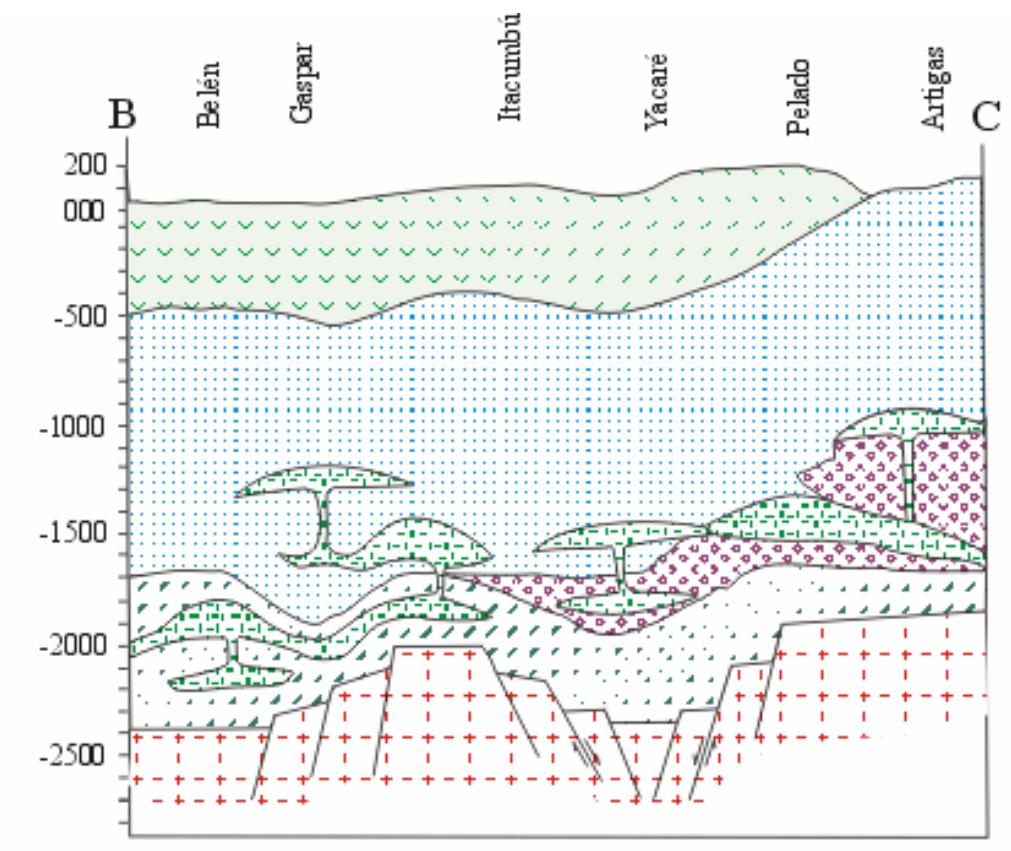

Corte B-C (W-E)

Escala horizontal aproxima da: $1: 100.000$

Escala vertical:

Los caudales de surgencia de las perforaciones en la zona norte:

\begin{tabular}{l|c|c}
\hline $\begin{array}{c}\text { Pozos } \\
\text { Termales }\end{array}$ & $\begin{array}{c}\text { Departament } \\
\mathbf{0}\end{array}$ & $\begin{array}{c}\text { Caudal } \\
\text { de } \\
\text { surgenci } \\
\mathbf{a} \\
\left(\mathbf{m}^{3} / \mathbf{h}\right)\end{array}$ \\
\hline Arapey & Salto & 340 \\
Damián & Salto & 134 \\
Hotel Quiroga & Salto & 230 \\
Ose & Salto & 90 \\
Club Remeros & Salto & 135 \\
Guaviyú & Paysandú & 125 \\
San Nicanor & Paysandú & 120 \\
\hline
\end{tabular}

\subsubsection{Zona Sur}

Hacia el sur (límite de la cuenca) se adelgaza el SAG típico y comienzan a observarse pozos (Guaviyù) que captan ambos sistemas, igualmente con predominio de las características hidráulicas y químicas del SAG. En este caso existe una conexión artificial efectuada por el pozo que pone en contacto los dos sistemas acuíferos, aunque no se descarta una conexión natural.

$\mathrm{Si}$ los ejemplos detallados se comprobaran los sistemas se transformaría en un macrosistema con condiciones hidráulicas y químicas únicas.

En la zona de borde, afectada por una gran actividad téctonica, se presentan casi en la misma latitud dos casos diferentes:

a) predominio del sistema de origen pérmico, como el pozo de Almirón con solo20m de sedimentos de SAG típico, resultando un pozo de agua con bajo caudal $\left(80 \mathrm{~m}^{3} / \mathrm{h}\right)$ y alto contenido en sales

b) Hacia el oeste a una distancia de $60 \mathrm{~km}$ en la ciudad argentina de Colón, se capta agua de un intertrap dentro del basalto con características acuíferas del SAG típico.

Para los casos de predominio del sistema de origen pérmico la transmisividad es del orden de los $50 \mathrm{~m}^{2} /$ día y el agua se clasifica hidrogeoquimicamente como clorurada sódica. 


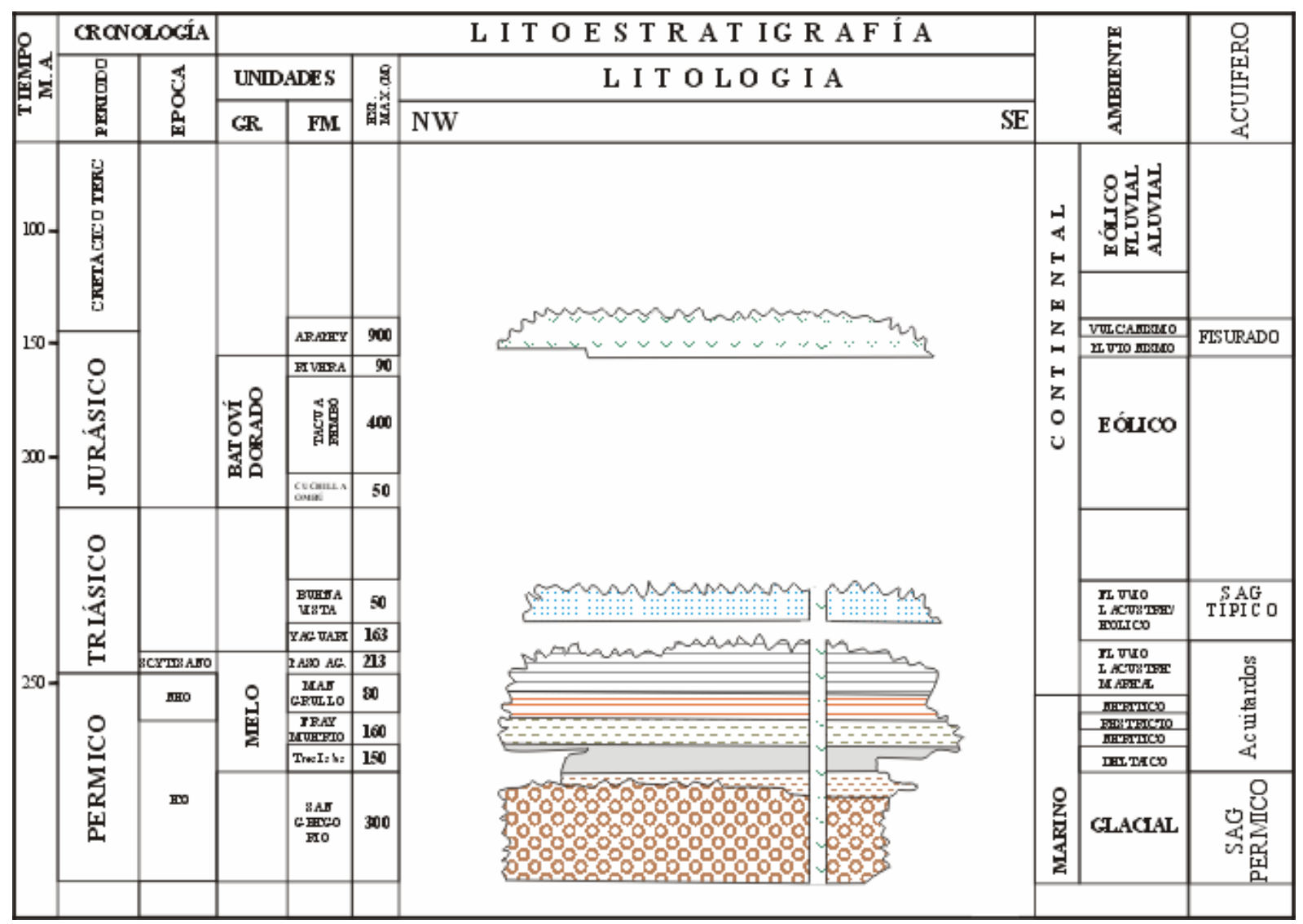

Figura 7. Columna estratigráfica Zona Sur. Sistema Acuífero Guaraní - Area Uruguaya

\subsection{Zona aflorante}

Ocupa un área de $3700 \mathrm{~km}^{2}$ en una faja de dirección N-S con un largo de 160 km y un ancho medio de $35-40 \mathrm{~km}$. Al interpretar hidrogeológicamente el subsuelo de la región se puede realizar la siguiente división:

\begin{tabular}{c|c|c|c}
\hline Grupo & Formación & Litología & $\begin{array}{c}\text { Capacidad del } \\
\text { acuífero }\end{array}$ \\
\hline \multirow{2}{*}{$\begin{array}{c}\text { Batoví } \\
\text { Dorado }\end{array}$} & Rivera (Botucatu) & $\begin{array}{c}\text { - areniscas finas a } \\
\text { medias }\end{array}$ & Bueno \\
\cline { 2 - 4 } & $\begin{array}{c}\text { Tacuarembó } \\
\text { (Piramboia) }\end{array}$ & - areniscas y pelitas & Regular \\
\hline
\end{tabular}

Este paquete sedimentario del Grupo Batoví Dorado se caracteriza por una sucesión de sedimentos de diferente permeabilidad, que lleva a clasificarlos en acuíferos con alta permeabilidad (Formación Rivera) y de baja permeabilidad (Formación Tacuarembó). En función de esta división se clasifica la región en:

\subsubsection{Area Rivera}

Se desarrolla el Sistema Acuífero con un predominio de sedimentos de origen eólico, presentando en esta zona los mayores espesores. La transmisividad ( $\mathrm{T}$ ) para el acuífero Rivera tiene valores de $150 \mathrm{~m}^{2} /$ día. Estos fueron obtenidos a través de ensayos de bombeo, realizados en la ciudad de Rivera en pozos de abastecimiento público (OSE), cuyos resultados se resumen a continuación:

\begin{tabular}{c|c|c|c|c}
\hline$N^{\circ}$ de Pozo & Prof. & Acuífero predominante & $\begin{array}{c}\mathrm{T} \\
\left(\mathrm{m}^{2} / \mathrm{día}\right)\end{array}$ & $\begin{array}{c}\mathrm{Q} \\
\left(\mathrm{m}^{3} / \mathrm{h} / \mathrm{m}\right)\end{array}$ \\
\hline $724 / 1$ & 84,50 & Rivera & 155 & 3,43 \\
\hline $961 / 1$ & 102,50 & Rivera & 151 & 3,44 \\
\hline 1161 & 51,30 & Rivera & 140 & 2,7 \\
\hline
\end{tabular}


El valor del coeficiente de almacenamiento $S$ es $10^{-3}$, indicativo del semiconfinamiento del acuífero.

Los valores del caudal específico (q), son del orden de $3 \mathrm{~m}^{3} / \mathrm{h} / \mathrm{m}$ y los rendimientos de los pozos varían entre 50 y $110 \mathrm{~m}^{3} / \mathrm{h}$.

La clasificación del agua es clorurada cálcica.

\subsubsection{Area Tacuerembó}

El sistema que predomina es el acuífero Tacuarembó, constituido por areniscas y pelitas de origen fluvial. La explotación del acuífero es baja debido fundamentalmente a su bajo potencial, por presentarse predominantemente los sedimentos finos. La profundidad media de los pozos en la zona es de $80 \mathrm{~m}$ y un caudal medio de $3,5 \mathrm{~m}^{3} / \mathrm{h}$; el caudal específico medio es de $20 \mathrm{~m}^{3} / \mathrm{dí} / \mathrm{m}$. Los valores de transmisividad son del orden de $25 \mathrm{~m}^{2} /$ día. La clase de agua en función de la composición química es bicarbonatada cálcica.

\section{Conclusiones}

Como puede observarse existe una gran variedad respecto a la geometría y composición del SAG tanto en la zona aflorante, como confinada que tiene su incidencia en las características hidráulicas y en la calidad de las aguas subterráneas.

Sus variaciones se deben principalmente a los fenómenos tectónicos como presencia de fallas, filones diabásicos, levantamientos, etc que condicionan el almacenamiento y circulación del agua subterránea marcando la gran heterogeneidad del sistema.

Esta situación es detectada en un área muy particular por su estructura al ser borde de una cuenca sedimentaria; influyendo en la heterogeneidad de los cuerpos permeables que constituyen el SAG. Estas características determinan una gama de variaciones en las características hidráulicas que se reflejan en la transmisividad con valores de 25 a $155 \mathrm{~m}^{2} /$ día para la zona aflorante y 300 a $400 \mathrm{~m}^{2} /$ día en la zona confinada. También existe una gran variedad de tipos de agua desde potables bicarbonatadas cálcicas a sódicas hasta clorurada sódicas, estas últimas influenciadas por el acuífero pérmico. Además existe gran variación en el caudal de surgencia de los distintos pozos, presentando valores que van desde 30 a $340 \mathrm{~m}^{3} / \mathrm{h}$.

Estos resultados demuestran que el SAG no constituye un sistema homogéneo y la seudo homogeneidad que se presenta en muchas publicaciones principalmente de simulación se debe simplemente a zonas con falta de información 0 de estudios detallados.

La información sobre el conocimiento de cualquier acuífero tiene una importancia socioeconómica para los Países porque se está caracterizando un recurso económico donde se marcan las pautas para su explotación y gestión racional. Es por ello que creemos que debe seguirse un camino lógico en el desarrollo del conocimiento partiendo de los estudios básicos hasta alcanzar los modelos conceptuales hidrogeológicos para luego sí poder simular cada situación y escenario.

\section{Bibliografia}

ANCAP, 1991. Estudio del Sector Noreste de la Cuenca Norte, Montevideo, Uruguay.

Goso H y H De Santa Ana, 1986. Estratigrafía de la Cuenca Norte del Uruguay. ANCAP.

Montaño J y R Carrión, 1990. Optimización de la Explotación del Sistema Acuífero Triásico Jurásico Tacuarembó - Buena Vista. Actas del $6^{\circ}$ Congreso de Aguas Subterráneas. Vol. I, 180-189, Porto Alegre, Brasil.

Montaño J y otros, 1999. Sistema Acuífero Guaraní. Capítulo Argentino Uruguayo. Universidad del Litoral, Argentina.

Tahal, 1986. Proyecto agrícola de Riego basado en la perforación de pozos profundos. Tahal Consulting Engineers VERNO. ROU, 8 tomos.

Veroslavsky, G, 1995. Análise Faciológica e Estratigráfica o Devoniano da Borda Sdul da Bacia do Paraná, 
Uruguay. Tesis de Maestría. Río Claro (SP), UNESP.

Montaño J. "Características hidráulicas e hidrogeoquímicas del acuífero Tacuarembó". Rev. A.B.A.S. (Rivera). Uruguay. 1987.

Montaño J. y otros "Características Hidráulicas e Hidrogeoquímicas del Acuífero Tacuarembó - Area Rivera". Pag 67-74. Revista de la Asociaçao Brasileira de Àguas Subterrâneas (ABAS). In 12 Ago 1988. Editorial: ABAS, Brasil.

Montaño J., Collazo P. "Vulnerabilidad del techo del SAG en la cuenca NW del Uruguay" $9^{\circ}$ Congreso Brasilero de Aguas Subterráneas. 25 al 28 de agosto de 1996 Salvador Bahía, Brasil.

Montaño J, Collazo P. "Hidrogeoquímica del Sistema Acuífero Guaraní (Uruguay)". II Congreso Uruguayo de Geología, Punta del Este, Uruguay, 1988.

Montaño J. y otros: "Informaçoes básicas sobre a distribuiçao do Sistema Aqüifero Guarany nas regiõnes Sul e Sudeste do Brasil". Pag 23-26. Compartido. A Água Em Revista Revista técnica e informativa da CPRM Servicio Geológico do Brasil. Año IV No 10 Junio de 1998. Editorial: CPRM Serviço Geológico do Brasil Divisao Marketing e Divulgaçao. Brasil.

Montaño J y otros. "El Acuífero Guaraní.
Un recurso compartido por Argentina, Brasil, Paraguay y Uruguay (América del Sur)". Pag 73-78. Compartido. Boletín Geológico y Minero. Hidrología Subterránea. Revista Bimestral del Instituto Tecnológico Geominero de España. Vol 109, № 4. Julio - Agosto de 1998. Editorial ITGE. España.

Montaño J., Collazo P., De Coud P. "Característica de la Evolución del Sistema Acuífero Guaraní en el Uruguay." $10^{\circ}$ Congreso Brasilero de Aguas Subterráneas. 9 al 11 de setiembre de 1998. San Pablo, Brasil.

Rosa Filho, E.F. da; Hindi, E.C.; Giusti, D.A.; Mantovani, L.E. 2000. Usos Potenciais do Hidrotermalismo do Sistema Aqüífero Guarani. Boletim Paranaense de Geociências, Curitiba. V. 48: p. 63-69.

Rosa Filho, E.F. da; Hindi, E.C. 2001. Structural features and its consequences in the Guarani aquifer system. In: International Conference on Hydrological Challenges in Transbondary Water Resources Management. Koblenz-Alemanha, Anais. Bundesanstalt fuer Gewaesserkunde Federal Institute of Hydrology. p. 1-8.

Rosa Filho, E.F. da; Hindi, E.C.; Giusti, D.A.; Nadal, C.A.; Xavier, J.M. 2001. Distribuição do Geotermalismo na Bacia Sedimentar do Paraná. Revista Latinoamericana de Hidrologia para el Desarrollo. Curitiba, Brasil. Imprensa Universitária da UFPR. № 1: p.67-74. 